\title{
Description of a restricted randomization program (RRP)*
}

\author{
NORMAN W. BRAY $\div$ and MARTIN D. MURPHY \\ Universitv of Illinois. Champaign-Lrbana \\ Champaign. Illinois 61820
}

A computer program, which produces restricted randomizations of numbers as an aid in counterbalancing large experimental designs, is described. The input to the program is a series of specifications for sampling a pool of random numbers. For example, it might be specified that each number in the pool be selected at least once and not more than twice in each replication of the experiment. The output would be a restricted randomization meeting these specifications. The program has been used in several memory experiments and could be used in other experimental paradigms in learning and perception.

This paper describes a computer program which generates restricted randomizations of numbers useful in counterbalancing large experimental designs. The program was originally developed to deal with counterbalancing problems in serial memory experiments (Bray. 1972. 1973). It soon became apparent that the general types of counterbalancing problems in serial memory experiments are similar to those in many areas of experimentation (e.g.. recognition memory. paired-associate learning, free recall. discrimination learning. concept formation. absolute judgment, backward masking. and others). Since then, the program has been used in several recognition memory experiments (Murphy. 1972: Murphy \& Scott, 1972).

In the recognition memory experiments, the $S$ is shown a set of to-be-remembered pictures followed by a retention interval and then a memory probe to which a "same" (appeared in the set) or "different" (did not appear in the set) response is made. A relatively small number of repeatedly used stimuli make up the memory sets and serve as probes for each trial. In this type of experiment. confounding stimuli with experimental variables such as retention interval, serial position, or type of probe must be avoided since the stimuli may vary in the ease with which they are remembered. Two methods are potentially useful in solving such confounding problems.

One solution, and possibly the most ideal, is to assign stimuli randomly to serial position, retention intervals, and probe types with a different randomization for each $S$. If words are used as stimuli and if on-line computing facilities are available. this solution is feasible. However,

*This project was supported by Research Grant USPH HD 05951 . Training Grant HD 00244. The Graduate College of the University of Illinois. HEW Division Maternal and Child Health Project Nos. 912 and 918, and the Department of Psychology, University of Cincinnati.

tRequests for reprints should be sent to Norman W. Bray, Department of Psychology, University of Cincinnati, Cincinnati, Ohio 45221. An annotated copy of RRP is available upon request. if the researcher either does not have access to on-line facilities or uses pictorial stimuli that require slide presentation. the use of a separate randomization for each $S$ becomes cumbersome. Under this set of circumstances. the use of restricted randomization for all Ss may be more reasonable. For instance. each stimulus might be used equally often at each level of serial position, retention interval, and probe type, and. where possible. equally often at all combinations of these variables.

The restricted randomization procedure, while providing an alternative to complete randomization, presents practical problems for large experiments. If done by hand, a table of random numbers must be consulted and the number of times each stimulus is used with each variable and combination of variables must be tallied. Such bookkeeping tasks are handled quite well by a computer. This paper describes a FORTRAN program developed for those purposes. The input to the restricted randomization program (RRP) includes the number of variables and levels of each, along with restrictions to be placed on the randomization. The output is an ordering of stimuli which meets the specifications.

The operation of RRP will be illustrated with a relatively simple example. In the recognition memory paradigm described earlier, assume that on each trial six different pictures are presented for study. When tested. the S must quickly respond "same" or "different." depending on whether the test picture was in the set. The program chooses which test pictures will be used on "different" trials. These are to be selected from a pool of nine and balanced so that each is used exactly twice in a given session (block) and not more than once in any one of three replications within a block. There are 12 blocks ( 36 trials per block). 12 trials per replication. and 6 "different" trials per replication.

The program solves this problem by selecting a random number for each trial and subjecting the number to specified tests. The program begins with the first trial of the first replication of the first block and proceeds through successive trials within each replication of each block. For this particular problem, a random number between 1 and 9 (corresponding to the nine pictures) is selected on each trial and subjected to two interrogative tests: (1) Has this number been selected during this replication? and (2) Has this number been selected twice within this block? If the answer is no, the number is "accepted" and stored. If the answer is yes. another random number between 1 and 9 is selected without replacement. If all nine numbers fail the test on Trial $n$. the program "backs up" to Trial $\mathrm{n}-1$. places the item selected on $\mathrm{n}-1$ into a temporary store. and tries to find a new solution. After this. the program again attempts to find a solution for Trial $n$. The recursive 


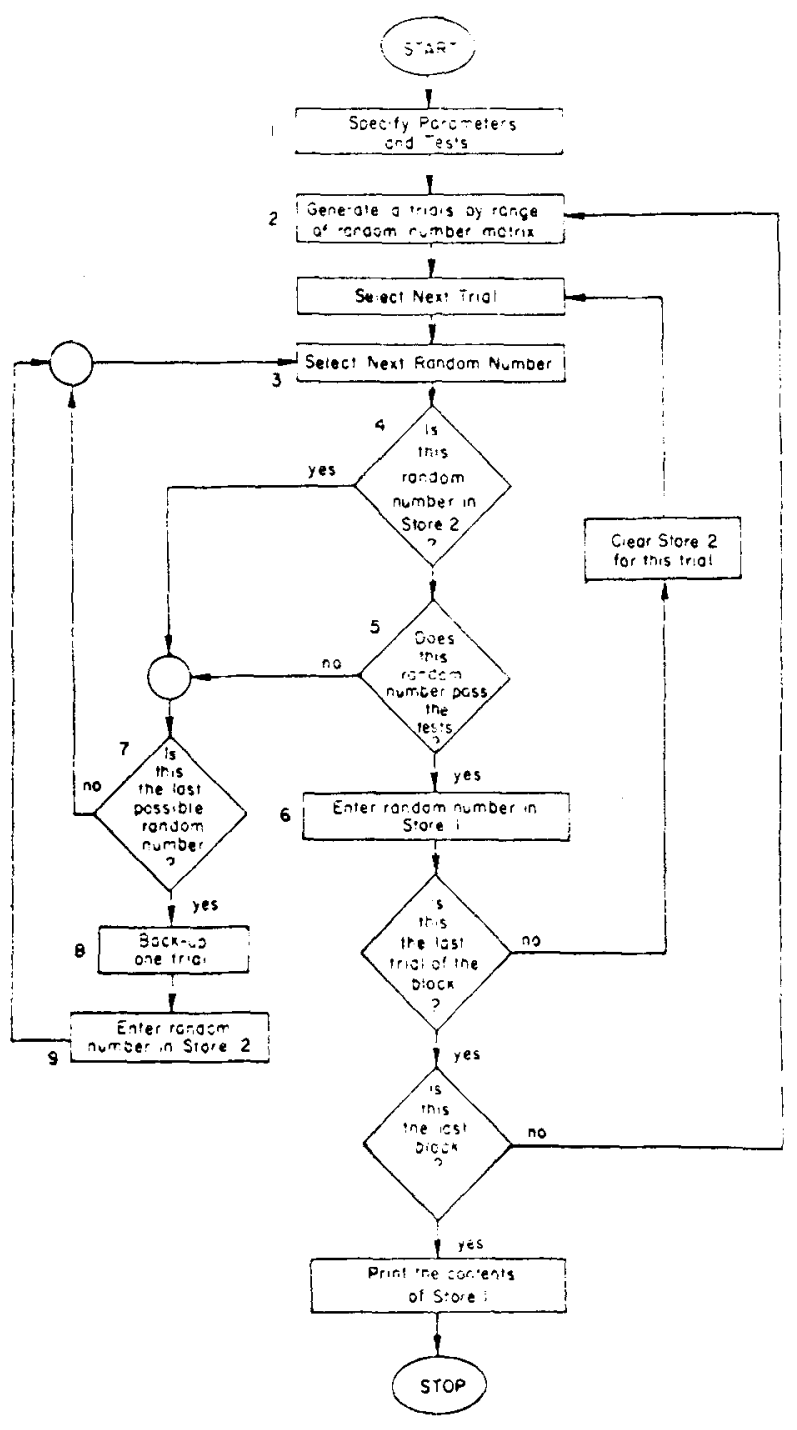

Fig. 1. Generalized flow chart for RRP.

nature of the search is used to correct for "bad runs" of randomization. so that the sequence selected meets the criteria specified. 1 The operation of the program will now be described in more detail.

\section{DESCRIPTION OF THE PROGRAM}

The description of RRP follows the general flow diagram shown in Fig. 1. The numbers in parentheses in the text correspond to the numbers in the figure.

To begin, the user must specify the size of the item pool, the number of trials within a block. the number of blocks, the number of tests. and the variables involved in each test (1). With this information. RRP generates a matrix of random numbers with all items in the pool sampled without replacement for each trial (2). Each column of the random number matrix corresponds to one trial in the block. After updating the trial number. the first number in Column $n$ of the random number matrix is selected as the item to be tested (3).

If the random number passes a check on whether or not it had been rejected previously on Triai $n$. it is subjected to the tests (5). The test mechanism consists of tally matrices that correspond to restrictions placed on the randomization. In the sample problem, a tally matrix would be constructed to correspond to the question, "Has the number been used before in this day?" This matrix would be a nine-element array. with one cell for each of the nine stimuli. When a random number is tested, the value in the corresponding cell is checked against a computed maximum. If the value is less than the maximum, the number passes the test. If the tally value is equal to the maximum, the number fails the test.

For the second restriction in the sample problem, a two-way tally matrix would correspond to the specification that no number be selected more than once within each replication. The 3 by 9 tally matrix would have one level for each of the three replications and one level for each of the nine stimuli. The testing procedure proceeds by comparing the entered value for a cell with the computed maximum.

If the number passes these tests, it is entered in Store $1(6)$. a check is made on the trial number, and the selection process moves on to the next trial. If any of the tests are failed, a check is made to see if all the random numbers in the pool have been tried for Trial $n$ (7). If not. the next number in the column for Trial $\mathrm{n}$ is called and tested. This cycle continues until all available numbers are tested or until an acceptable number is found. In the event that an acceptable number cannot be found. and the number of alternatives have been exhausted, RRP "backs up" to Trial $n-1$ (8). places the number previously accepted on Trial $n-1$ into Store 2 (9), and begins the selection for Trial $\mathbf{n}-1$ again (3). When a number is chosen for Trial $n-1$. it is checked against the contents of Store 2 to prevent its being accepted again (4).

The search on Trial $n-1$ continues in this manner until an acceptable random number has been found or all possibilities have been tested and found unacceptable. If the search is successful. RRP proceeds to Trial $n$ and attempts another solution, beginning with the first random number for that trial. If the search is unsuccessful on $n-1$, the same process is repeated for Trial $\mathrm{n}-2$. etc. If RRP tries to back up beyond the first trial of a block. the information in Store 1 for the previous block is cleared, and the search started on Trial 1 of that block. The user may also specify the number of back-ups within a block permissible before backing up to the first trial of a block to attempt a new solution for the whole block.

When a solution for all trials of a block has been found. RRP creates a new matrix of random numbers and begins to search for a solution for the first trial of the next block. When the last trial of the last block is solved. RRP prints the solution for all trials for all blocks and stops.

RRP consists of MAIN and three subroutines 


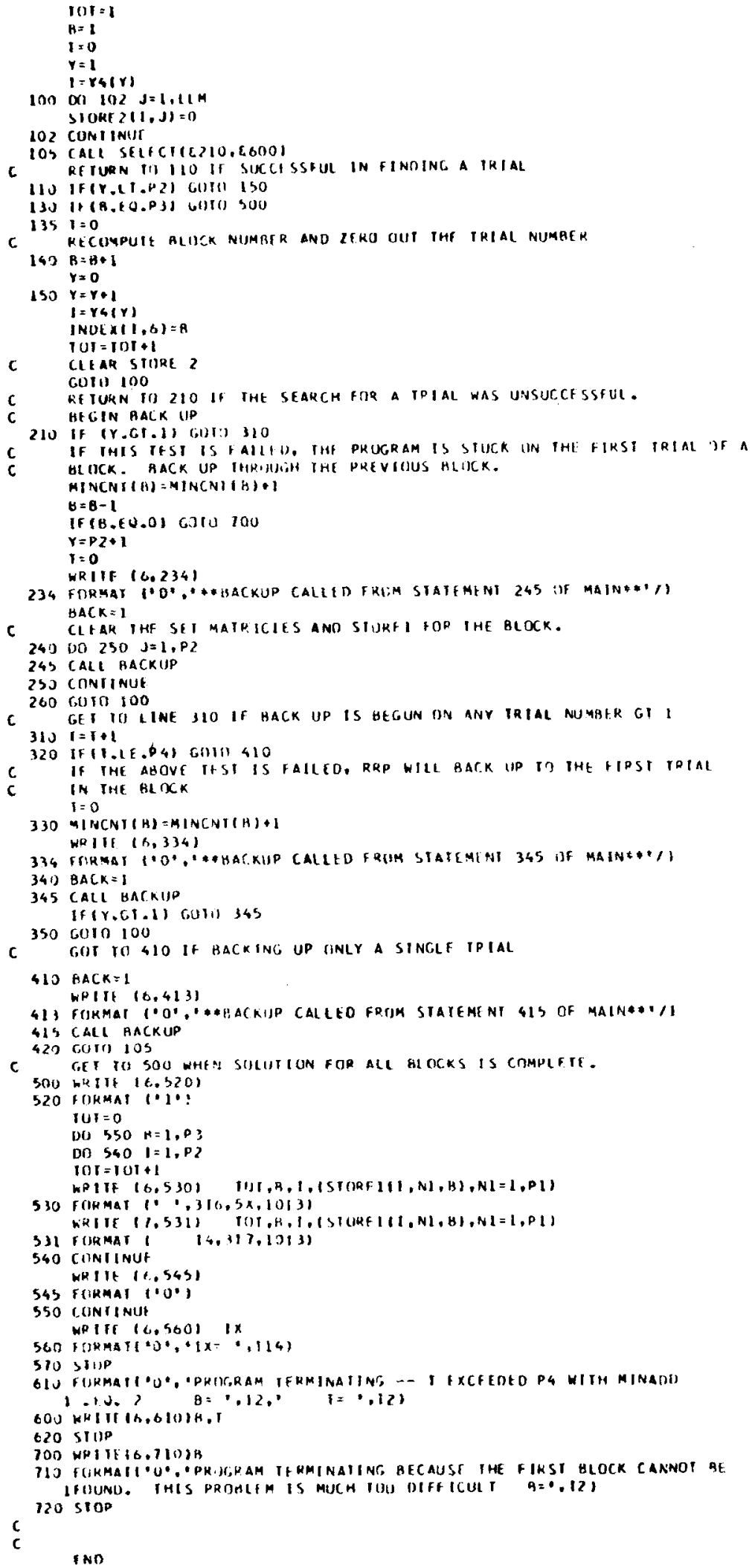

Fig. 2. (A) FORTRAN listing of RRP. 


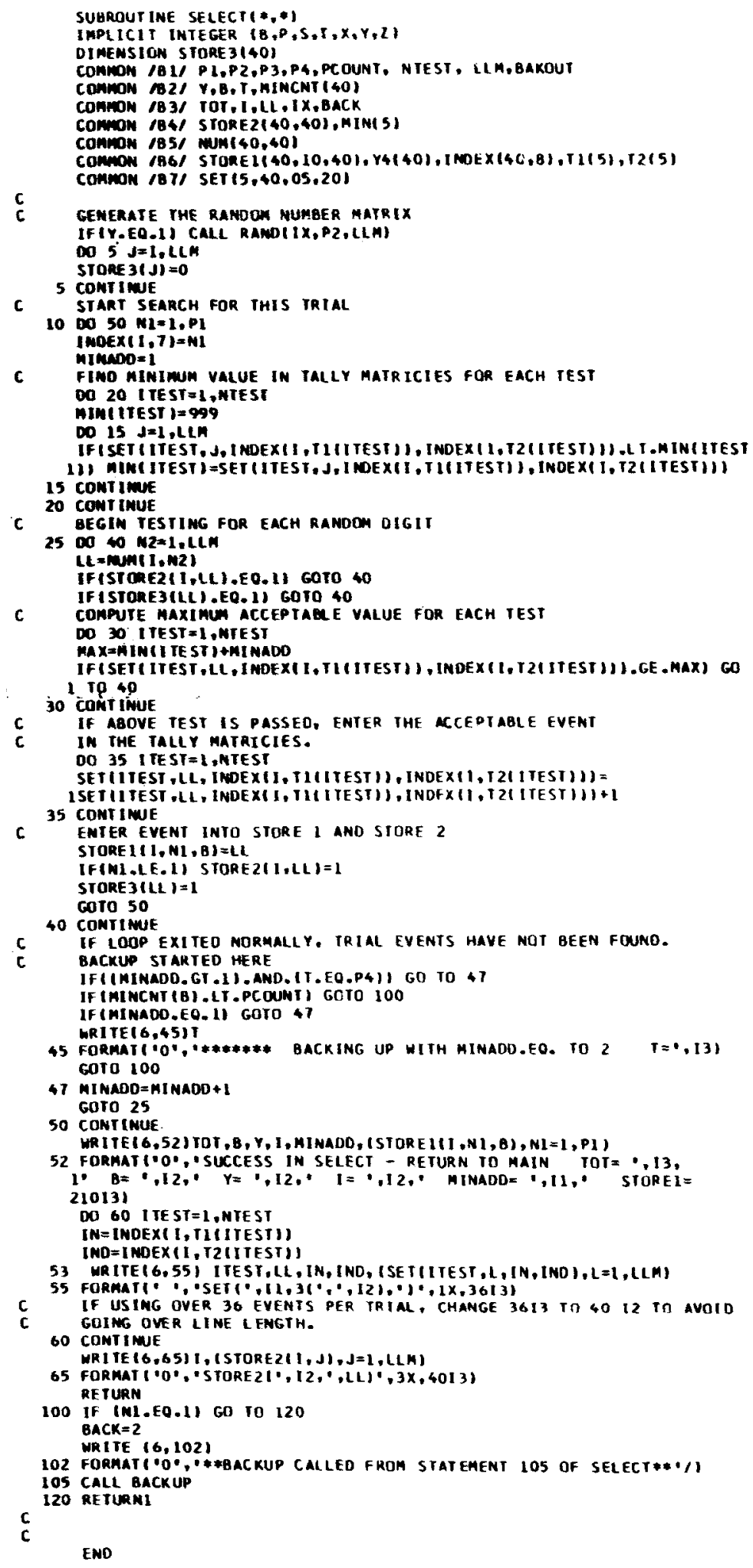

Fig. 2 (B)

SELECT, BACKUP, and RAND. A FORTRAN listing of a segment of MAIN and the three subroutines in their entirety is shown in Fig. 2. MAIN monitors the search, initiates a back-up, and prints the final solution. SELECT does the testing within the tally matrices (Set) and BACKUP clears the necessary information from Set 


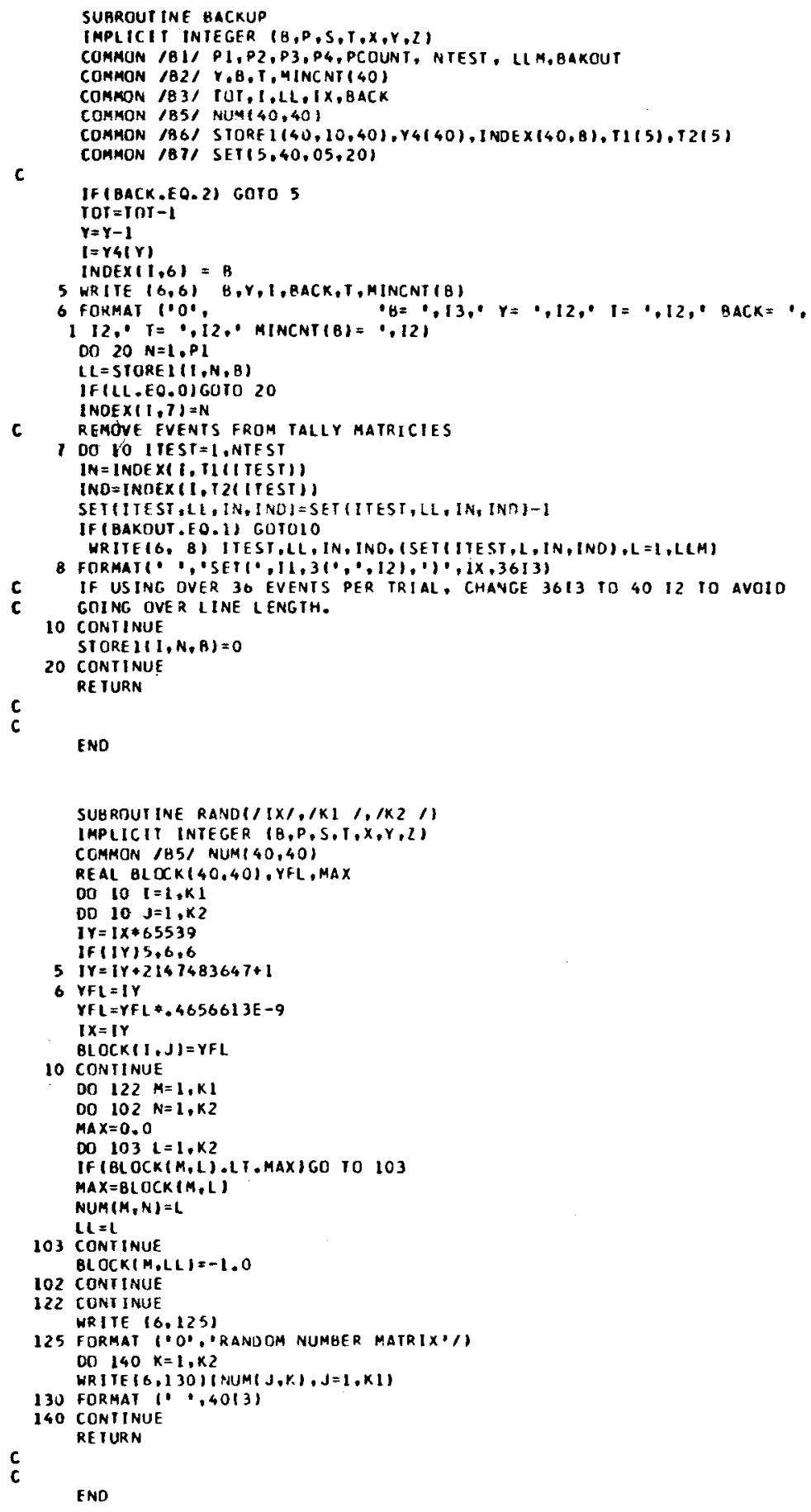

Fig. 2 (C)

and Store 2 when RRP backs up. RAND generates the random number matricies. This subroutine is constructed around RANDU, which is taken from IBM's (1969) Scientific Subroutine Package. RANDU generates pseudorandom numbers between 0.00 and 1.00 on a cycle which repeats itself only after $2^{29}$ events. The cycle is entered at different points by specifying a random odd integer of seven to nine digits. RAND converts the output of RANDU to integers of the specified range. The characteristics of RANDU minimize the similiraty of the solution obtained for each trial within a block, and for each block within the experiment. A sample solution for the example described in the text is shown in Fig. 3.

\section{APPLICATIONS, OPTIONS, AND RESTRICTIONS}

In several recognition memory experiments (Murphy, 1972; Murphy \& Scott, 1972), RRP has solved problems 


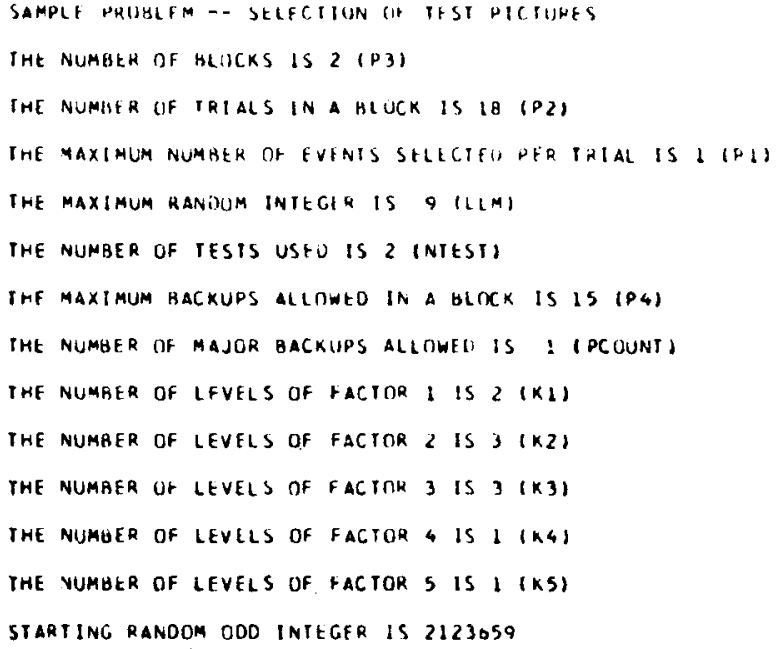

SOLUT IGN

\begin{tabular}{|c|c|c|c|c|c|c|c|c|}
\hline IRIALS & BLOCK & IYPE & EVENTS & & ELE & TE & & \\
\hline 1 & I & 1 & 3 & 6 & 7 & 1 & 9 & 2 \\
\hline 2 & I & 2 & 8 & 9 & 3 & 5 & 6 & 4 \\
\hline 3 & $i$ & 3 & 9 & 5 & 6 & 1 & 8 & 7 \\
\hline 4 & 1 & 4 & 1 & 2 & 3 & 8 & 4 & 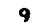 \\
\hline 5 & 1 & 5 & 4 & 2 & 3 & 5 & 9 & 7 \\
\hline 6 & i & 6 & $i$ & 5 & 2 & 1 & 9 & B \\
\hline 7 & 1 & 7 & 4 & 6 & 9 & 2 & 3 & I \\
\hline 8 & 1 & 8 & 7 & 6 & 3 & 1 & 2 & 4 \\
\hline 9 & 1 & 9 & 4 & 8 & 5 & $i$ & 9 & 7 \\
\hline 10 & 1 & 10 & 7 & 5 & 6 & $i$ & 4 & 9 \\
\hline 11 & 1 & 11 & 4 & -2 & 6 & 8 & 5 & 3 \\
\hline 12 & 1 & 12 & 2 & 3 & 8 & 5 & 6 & 7 \\
\hline 13 & $i$ & 13 & 5 & 1 & 7 & 3 & 2 & 9 \\
\hline 14 & $i$ & 14 & 6 & 4 & 1 & 2 & 5 & 8 \\
\hline 15 & i & 15 & 6 & 8 & 4 & 7 & 1 & 5 \\
\hline 16 & 1 & 16 & 3 & 9 & 7 & 1 & 6 & 5 \\
\hline 17 & 1 & 17 & 9 & 3 & 6 & 8 & 4 & 2 \\
\hline 18 & 1 & 18 & B & 9 & 2 & 7 & 3 & 4 \\
\hline 19 & 2 & 1 & 3 & 4 & 7 & 9 & 5 & 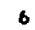 \\
\hline 20 & 2 & 2 & 4 & 2 & 5 & $i$ & $i$ & 8 \\
\hline 21 & 2 & 3 & 1 & 8 & 2 & 7 & 6 & 9 \\
\hline 22 & 2 & 4 & 3 & 6 & 9 & 5 & B & 1 \\
\hline 23 & 2 & 5 & 4 & 3 & 2 & $B$ & 5 & 9 \\
\hline 24 & 2 & 6 & 9 & 3 & 6 & 7 & 4 & 2 \\
\hline 25 & 2 & 7 & 1 & 7 & 8 & 6 & 5 & 2 \\
\hline 26 & 2 & 8 & 9 & 1 & 5 & 8 & 6 & 7 \\
\hline 27 & 2 & 9 & 1 & 4 & 3 & 9 & 6 & 5 \\
\hline 28 & 2 & 10 & 2 & 3 & 4 & 6 & 1 & 9 \\
\hline 29 & 2 & 11 & 4 & $i$ & 3 & 8 & 7 & 6 \\
\hline 30 & 2 & 12 & 8 & 1 & 4 & 5 & 2 & 3 \\
\hline 31 & 2 & 13 & 2 & $i$ & 7 & 8 & 9 & 3 \\
\hline 32 & 2 & 14 & 4 & 9 & 1 & 6 & 2 & 5 \\
\hline 33 & 2 & 15 & 5 & 4 & 2 & 8 & 6 & 1 \\
\hline 34 & 2 & 16 & 7 & 8 & 5 & 9 & 4 & 6 \\
\hline 35 & 2 & 17 & 5 & 3 & 4 & 9 & 1 & 2 \\
\hline $3 E$ & 2 & 18 & 3 & 7 & 2 & 1 & 3 & \\
\hline
\end{tabular}

Fig. 3. Sample output from RRP.

in which the number of times a given picture is used as a stimulus picture and as a study picture, as well as the number of times it is used as a test picture, are balanced across days. RRP has solved other complex problems in recognition memory experiments, including the production of a restricted randomization such that each picture from three different functional categories were balanced within each session and across several other variables. In experiments on serial memory (Bray, 1972, 1973), the digits 1 through 9 were balanced within each of 10 sessions. In the balancing, each digit was used equally often as a test item, and occurred as a study item an equal number of times across days and trial types.

In some cases, the solutions required several runs, each run balancing a separate component of the design. For instance, in the recognition memory experiment 
using categorized pictures, one run selected the serial position to be tested on each trial, a second run selected test pictures for those positions, and a third filled in the remaining serial positions.

In solving some of these problems, several options have been developed. One option allows the selection of multiple events per trial, so that all pictures used as study stimuli on a given trial can be selected on one run. Another option allows for the specification of varying degrees of restrictiveness in the final solution. The researcher can specify a perfect solution for problems of low and medium difficulty, but allow an approximate solution with very difficult problems. A third option allows the researcher to determine how the program will operate when a back-up is required: either back up one trial at a time or, after a specified number of one-step back-ups, start over at the beginning of the block.

RRP, as listed in Fig. 2, will accept problems with up to 40 individual events to be randomized, 40 blocks, 40 trials per block, 10 events per trial, and 5 two-way tests. With these maxima, RRP requires approximately $200 \mathrm{~K}$ for the FORTRAN G compiler on the IBM 370/65 at the Southwestern Ohio Regional Computing Center (SWORCC). The storage space would be reduced considerably if the user reduced these maxima.

In order to illustrate the expected performance of RRP, a series of test runs were conducted. The parameters for these tests were chosen to span the parameter values we have typically used. All problems had five blocks, with three factors and three levels per factor, 27 trials per block. The parameters were a factorial combination of 1,2 , and 3 one-way tests, 1,5 , and 9 events per trial, and 12,18, and 30 events to be randomized. Each problem began with a different randomly selected seven-digit odd integer and was restricted to $30 \mathrm{sec}$ CPU time. The CPU times for these problems are shown in Table 1 . The tests were run on the IBM 370/65 at the SWORCC at the University of Cincinnati. Table 1 may be useful in estimating cost, since CPU time is a major expense at most university
Table 1

Test Run CPU Times (Seconds) on GO STEP of FORTRAN $G$ Compiler

\begin{tabular}{lccr}
\hline & \multicolumn{3}{c}{ Number of Events in Item Pool } \\
\cline { 2 - 4 } $\mathrm{N}^{*}$ & 12 & 18 & 30 \\
\hline & & One Test \\
3 & 3.05 & 1.80 & 2.53 \\
5 & 3.46 & 2.19 & 2.81 \\
& 3.72 & 2.52 & 3.16 \\
1 & & Two Tests & \\
3 & 5.26 & 2.81 & 4.27 \\
5 & 9.41 & 6.37 & 10.88 \\
& 9.72 & 5.96 & 11.48 \\
1 & & Three Tests & \\
3 & 4.30 & 4.72 & 4.78 \\
5 & 7.67 & 8.56 & 11.11 \\
\hline
\end{tabular}

*Number of events per trial

computer centers. The tests also provide some indication of "easy" and "hard" problems for RRP.

\section{REFERENCES}

Bray, N. W. The effect of instructions to forget on recall. Paper presented at the Gatlinburg Conference on Mental Retardation, March 1972, Gatlinburg, Tennessee.

Bray, N. W. Controlled forgetting in the retarded. Cognitive Psychology, 1973, 5, in press.

IBM. Scientific Subroutine Package. White Plains, N.Y: International Business Machines, Corp., 1969, Manual H20-0205-03.

Murphy, M. D. Categories in recognition memory. Paper presented at the Gatlinburg Conference on Mental Retardation, March 1972, Gatlinberg, Tennessee.

Murphy, M. D., \& Scott, K. G. Effects of category information on recognition memory in retardates. Unpublished manuscript, University of Illinois, 1972.

\section{NOTE}

1. It should be recognized that the more the restrictions put on the randomization, the more difficult the solution, and the user may overspecify the sequence so that no solution is possible.

(Received for publication January 11, 1973; revision received August 7, 1973.)

\section{ERRATUM}

Carlson, V. R., and Knight, I. SCV-A general subroutine for the selection of combinations of variables. Behavior Research Methods and Instrumentation, 1973, 5, 425-427-Table 2 should read as follows:

$\operatorname{ICND}(1)=1$

DO $100 \mathrm{~J}=1, \mathrm{NVAR}$

$100 \operatorname{ICND}(\mathrm{I})=(\operatorname{ICND}(\mathrm{l})-1) * \mathrm{NAIT}(\mathrm{J})+\operatorname{MALT}(\mathrm{J})$ 